\title{
Electromagnetic modelling of few-moded Winston cones in the far-infrared
}

Cite as: AIP Conference Proceedings 616, 295 (2002); https://doi.org/10.1063/1.1475647

Published Online: 06 May 2002

Emily Gleeson, J. Anthony Murphy, Sarah E. Church, Ruth Colgan, and Créidhe O'Sullivan
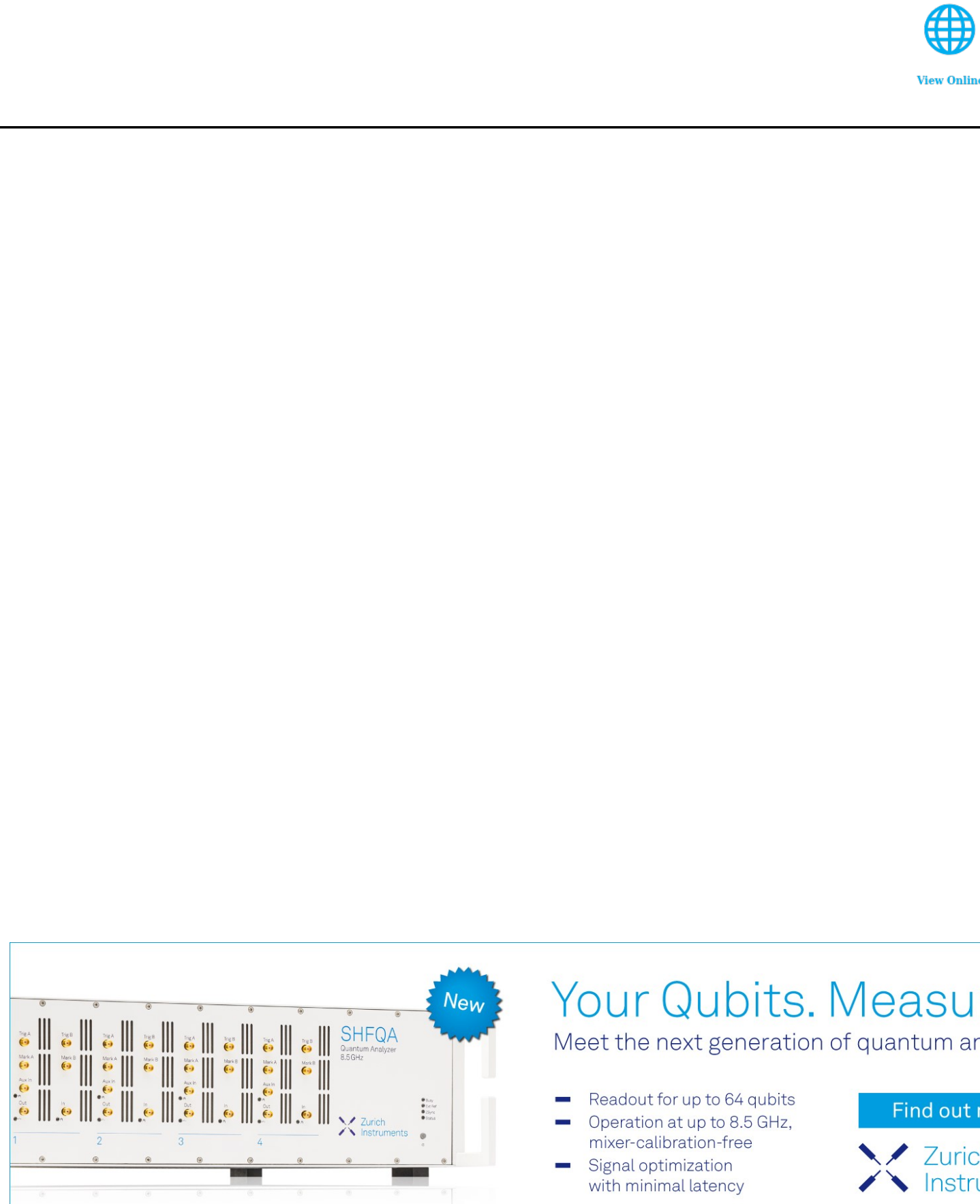

\section{Your Qubits. Measured.}

Meet the next generation of quantum analyzers

- Readout for up to 64 qubits

- Operation at up to $8.5 \mathrm{GHz}$.

- Signal optimization with minimal latency 


\title{
Electromagnetic Modelling of Few-Moded Winston Cones in the Far-Infrared
}

\author{
Emily Gleeson ${ }^{1}$, J. Anthony Murphy ${ }^{1}$, Sarah E. Church ${ }^{2}$, Ruth Colgan ${ }^{1}$, \\ Créidhe O'Sullivan ${ }^{1}$ \\ ${ }^{I}$ National University of Ireland, Maynooth, Co. Kildare, Ireland. \\ ${ }^{2}$ Stanford University, Stanford, CA 94305, USA.
}

\begin{abstract}
Winston cones have traditionally been used as detector feeds in far-infrared cosmological experiments, such as SuZIe, the Sunyaev-Zel'dovich Infra-red Experiment [1] on the CSO. They are usually designed using ray tracing, which becomes a very poor approximation when the number of spatial modes propagated by the horn is small in number, often the case at the longest wavelengths. We describe a more accurate approach involving electromagnetic modelling of Winston cones using a rigorous electromagnetic mode matching technique. It is straightforward to also consider the case of fewmoded corrugated Winston cones, which offer lower sidelobe levels than smooth walled cones which is important for high sensitivity experiments. Furthermore, the mode matching technique allows more complex structures such as back-to-back Winston cones and the detector cavities to also be analysed.
\end{abstract}

\section{INTRODUCTION}

A Winston cone is an off-axis parabolic profiled horn designed to maximise the collection of incoming rays within a field of view determined by its dimensions (see Figure 1). Equation 1 describes the Winston cone geometry where $z$ is the distance from the horn throat, $\rho$ is the horn radius and $d$ is the diameter of the horn throat. Equation 2 defines the maximum angle, $\alpha$, in the geometrical limit at which the incoming rays reach the exit aperture of the cone where $D$ is the diameter of the horn aperture.

$$
\begin{gathered}
z^{2}=\left(\frac{2 \rho+d}{2 d}\right)^{2}\left[(2 \rho)^{2}-d^{2}\right] \\
\sin (\alpha)=\frac{d}{D}
\end{gathered}
$$

Winston cones are usually modelled using ray tracing but this clearly becomes a poor approximation when few modes propagate. A mode matching technique can be used to achieve high accuracy and also enable complex structures like back-to-back Winston cones and detectors with cavities to be modelled. We also wish to consider the effect of corrugating the horn antenna to reduce sidelobe levels in an analogous way to a conical corrugated horn.

In the mode matching technique [2] the corrugated horn [3], [4] structure is regarded as a sequence of cylindrical waveguide segments with the radius stepping between the top and bottom of the corrugation slots. The smooth walled profile is approximated with a series of cylindrical monotonically increasing radii giving a stair like profile. The natural modes of propagation for each segment are the TE and TM modes of a uniform cylindrical waveguide. There is a sudden change in the guide radius at the interface between two segments and the power carried by the individual modes is scattered between the backward propagating modes in the first guide segment and the forward propagating modes in the second guide segment. The mode matching technique is based on matching the total transverse field in the two guides at the junction so that the total power is conserved and that the usual boundary conditions apply to the fields at the conducting walls. Track is also kept of the evanescent modes in the guide as these can propagate as far as the next corrugation. 


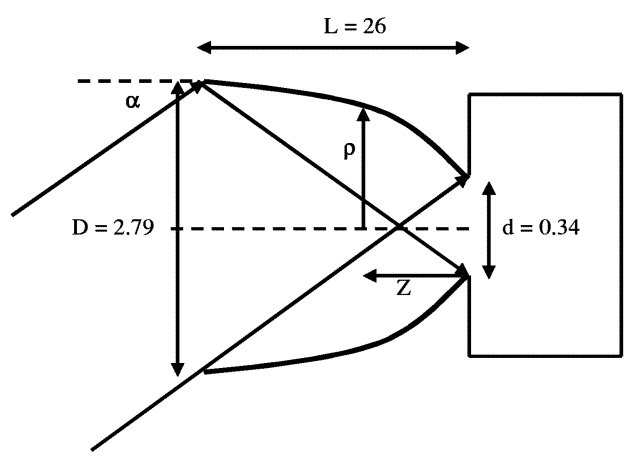

FIGURE 1. Schematic diagram of a Winston cone with a cavity.

The modal field at the aperture propagates to the farfield without scattering. If the waveguide filter is well matched to an integrating cavity, all the waveguide modes at the cavity entrance to the waveguide horn structure are assumed to be equally excited (carry an equal amount of power).

\section{EXAMPLES}

The farfield of a Winston cone is 'top-hat like' as a result of the extreme profile in its geometry. The design frequency used in the following examples is $545 \mathrm{GHz}$. In figure 2 the farfield patterns of different Winston cones (smooth walled and corrugated back-to-back cones and smooth walled and corrugated cones with a $4 \mathrm{~mm}$ cavity) are compared (dimensions as in Figure 1). The corrugated horns have a higher level of sidelobe rejection than the smooth walled horns. This is necessary for CMB experiments so that strong sources do not contaminate the results. The power pattern is in decibels to emphasise the sidelobe structure.

In figure 3 the frequency dependence of the farfield radiation patterns of some Winston cone geometries are compared. As the frequency decreases $(260 \mathrm{GHz})$ the horn becomes single-moded and as the frequency increases $(1000 \mathrm{GHz})$, more modes propagate and the beam becomes more top-hat like. At very high frequencies the farfield pattern resembles the geometrical beam for a smooth walled horn.

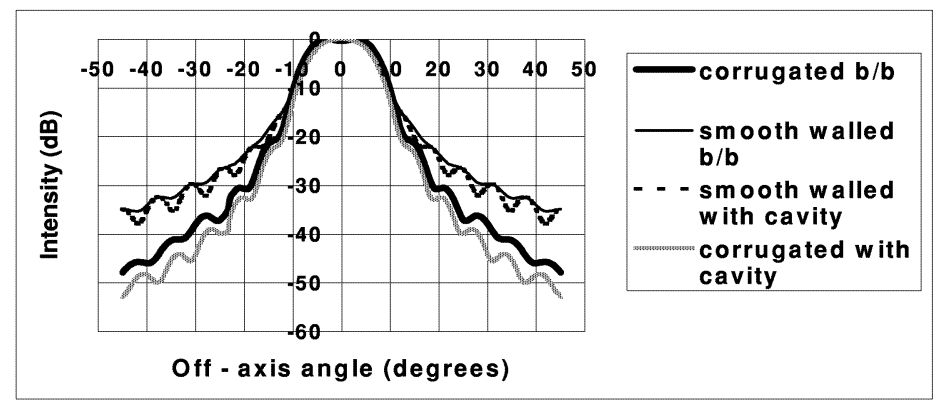

FIGURE 2. Farfield Radiation Patterns.

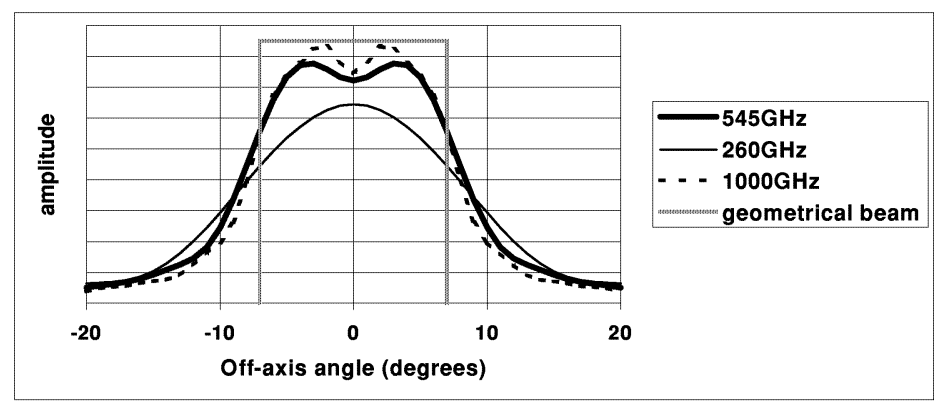

FIGURE 3. Frequency dependence of the farfield of Winston cones. 


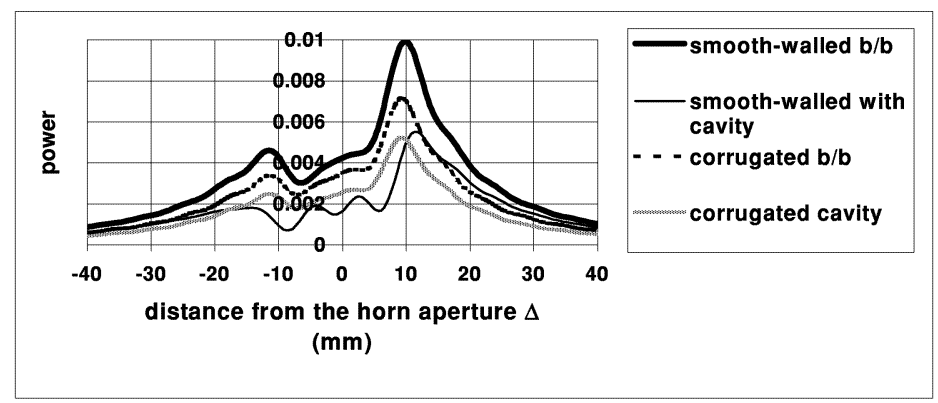

FIGURE 4. Phase-centre of Winston cones (negative $\Delta$ implies behind the aperture).

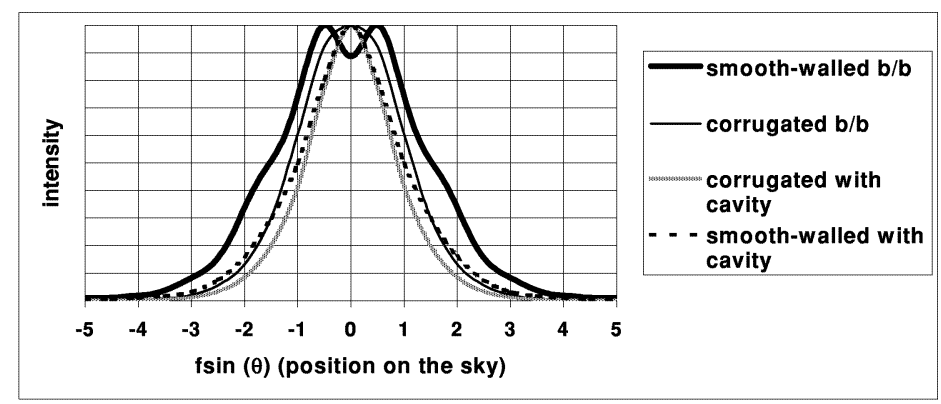

FIGURE 5. Radiation Patterns on the sky.

The phase-centre is determined by coupling the farfield radiation pattern of the horn to a telescope, for different axial positions, $\Delta$, of the horn, and then maximising the on-axis power (gain). This is illustrated in figure 4 with phase-centre values ranging from 9-12mm for the Winston cones in question (defined in Figure 1).

In an imaging telescope configuration the radiation pattern on the sky is obtained by fourier transformation of the partially spatially coherent farfield of the horn truncated at the telescope aperture, where the horn is positioned for maximum on-axis gain. Figure 5 illustrates these patterns for various Winston cones.

\section{ACKNOWLEDGEMENTS}

The authors would like to acknowledge the support of Enterprise Ireland who fund this research.

\section{REFERENCES}

1. http://www.astro.caltech.edu/ /gg/suzie/sins.html

2. Murphy, J.A., Colgan, R., O’Sullivan, C., Maffei, B., Ade, P., Infrared Physics and Technology, (in press, 2001).

3. Clarricoats P.J.B., and Olver A.D., Corrugated Horns for Microwave Antennas, Peter Peregrinus Ltd, London, 1984, (chapter 3).

4. Olver A.D., Clarricoats P.J.B., Kishk A.A. and Shafai L., Microwave Horns and Feeds, IEEE Press, 1994, (chapter 9). 